\title{
Corrigendum
}

\section{Corrigendum to "Probing for Sparse and Fast Variable Selection with Model-Based Boosting"}

\author{
Janek Thomas $\left(\mathbb{D},{ }^{1}\right.$ Tobias Hepp $\left(\mathbb{D},{ }^{2}\right.$ Andreas Mayr $\left(\mathbb{D},{ }^{2,3}\right.$ and Bernd Bischl ${ }^{1}$ \\ ${ }^{1}$ Department of Statistics, LMU München, München, Germany \\ ${ }^{2}$ Department of Medical Informatics, Biometry and Epidemiology, FAU Erlangen-Nürnberg, Erlangen, Germany \\ ${ }^{3}$ Department of Medical Biometry, Informatics and Epidemiology, University Hospital Bonn, Bonn, Germany
}

Correspondence should be addressed to Tobias Hepp; tobias.hepp@uk-erlangen.de

Received 10 May 2018; Accepted 16 May 2018; Published 5 July 2018

Copyright (C) 2018 Janek Thomas et al. This is an open access article distributed under the Creative Commons Attribution License, which permits unrestricted use, distribution, and reproduction in any medium, provided the original work is properly cited.

In the article titled "Probing for Sparse and Fast Variable Selection with Model-Based Boosting" [1], an Acknowledgment should be added as follows:

\section{Acknowledgments}

"Tobias Hepp performed the present work in partial fulfilment of the requirements for obtaining the degree 'Dr. rer. biol. hum' at the University of Erlangen-Nuremberg."

\section{References}

[1] J. Thomas, T. Hepp, A. Mayr, and B. Bischl, "Probing for sparse and fast variable selection with model-based boosting," Computational and Mathematical Methods in Medicine, Art. ID 1421409, 8 pages, 2017 


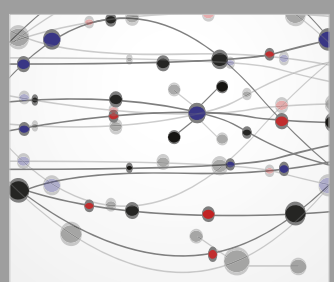

The Scientific World Journal
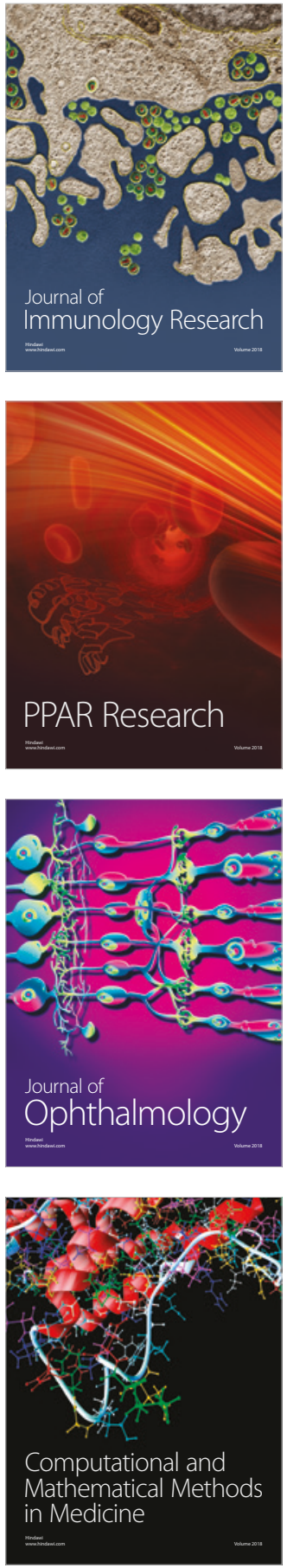

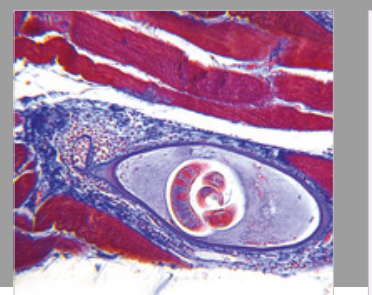

Gastroenterology Research and Practice

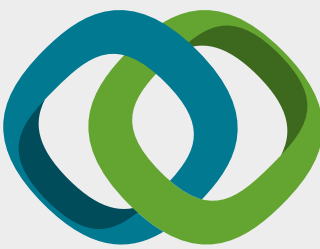

\section{Hindawi}

Submit your manuscripts at

www.hindawi.com
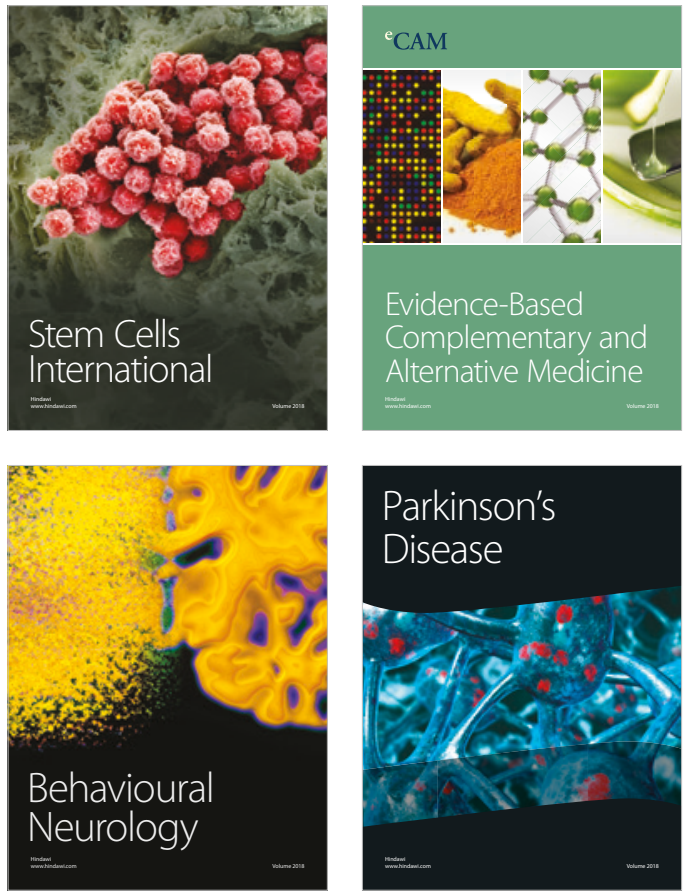

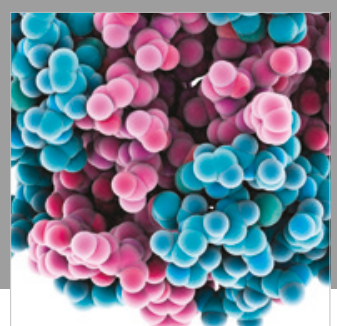

ournal of

Diabetes Research

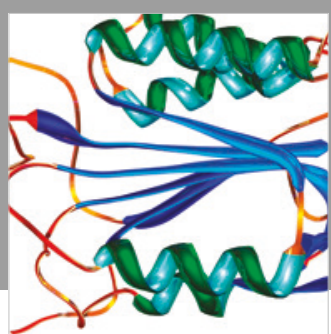

Disease Markers
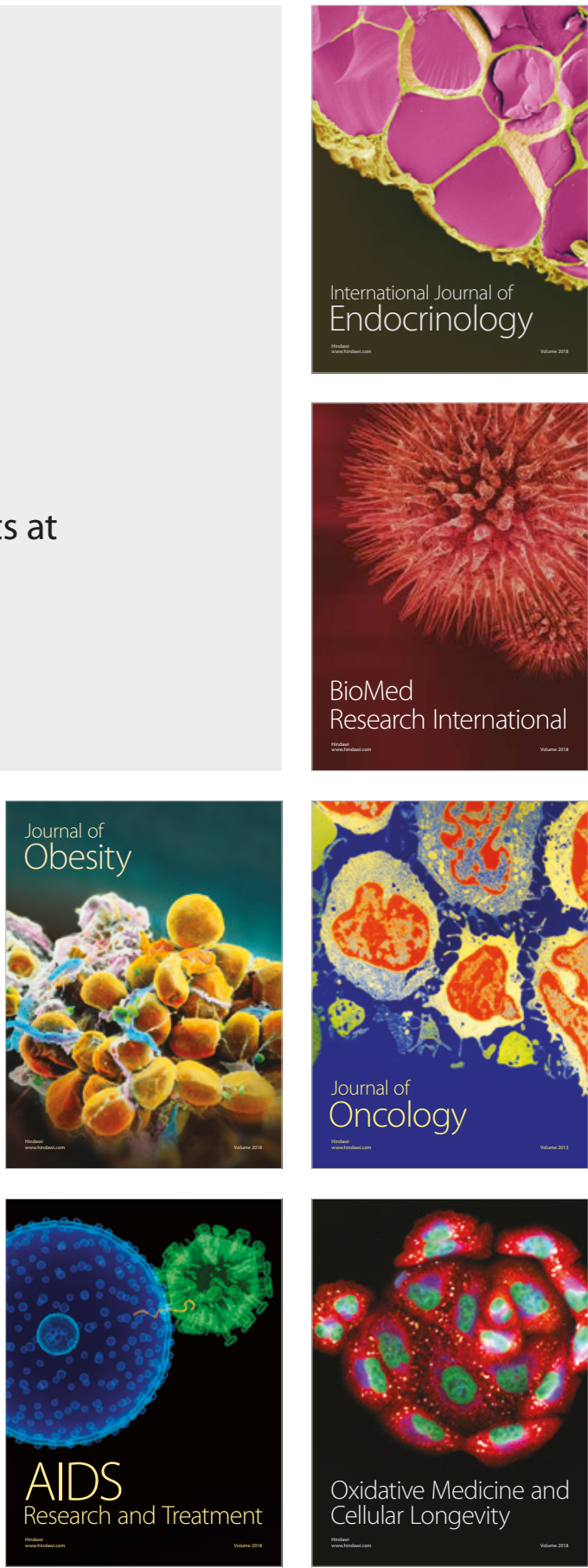